\title{
Management of Web Services Communities, WSC System
}

\author{
Victor Popa ${ }^{1}$, Liliana Constantinescu ${ }^{1}$, Victoriana Popa ${ }^{2}$, Maria Moise ${ }^{3}$, Carmen Rotună ${ }^{1}$ \\ 1 National Institute for Research and Development in Informatics - ICI, Bucharest, \\ 8-10 Averescu Avenue, Bucharest 1, România, \\ vpopa@ici.ro, lconst@ici.ro, karma_petcu28@yahoo.com \\ 2 Reply, 25 Cenisio Avenue, Milano, Italy, \\ vpopa.Consultant@ubm.unicredit.it \\ 3 Romanian-American University, \\ 1B Expoziției Avenue, Bucharest 1, România, \\ maria.moise@rau.ro
}

\begin{abstract}
The paper presents the system WSC, designed to provide modeling, organization, management and execution of Web services. The Service and Community ontologies are used for modeling of the Web services and Web services communities. Web Services are organized in semantic communities, in order to be easily accessed. Thus, the users of system navigate through the taxonomy of communities and invoke the target generic operations of communities. If an invoked operation requires some pre-operations to be executed before it, the system builds process diagram for these pre-operations. The user executes interactively each operation of diagram process using the interface provided by the system.
\end{abstract}

Keywords: community ontology, service ontology, registration of services with communities, process diagrams, invocation of community's generic operations, execution of Web service's methods.

\section{Introduction}

The number of Web services is increasing fast. The large scale and dynamics of these Web services hinders the understanding of their semantics and hence their management. To address this issue we propose the system WSC. Compared with other approaches which propose the ontologies for describing Web services (DAML-S, WSMF, OWL-S etc ) the WSC system provides means for both semantic description of Web services and their organization into communities.

The system WSC is the result of sustained research work on finding innovative and effective technologies for modeling, managing and accessing Web services.

To achieve this goal WSC system took into consideration both existing industry standards for modeling services (WSDL, SOAP, UDDI, BPEL) as well as academic initiatives in the area of semantic Web (DAML-S, WSMF etc).

The following requirements need to be considered when designing the WSC system:
- organizing web services space in semantic communities in order to effectively discover services

- understanding the capabilities of semantic web services

- discovering and accessing Web services using semantic criteria

- simple and fast designation of the processes used in invoking Web services methods

- using standard BPEL for composing Web services and communities

To meet the requirements listed above, WSC system provides the following features:

- Community [1][2] ontology, used as templates for creating community descriptions

- Service [2] ontology, used as templates for creating service descriptions

- WSDL-S formalism, used to create descriptions for communities and services, according to the two ontology

- communities taxonomy management

- Web services registration with communities taxonomy

- Web services discovery based on semantic criteria 
- mapping the generic operation invocations to Web service method invocations, based on quality attributes of Web services (response time, cost, reliability, confidentiality, etc.)

- automatic generation of process diagrams used to invoke generic operations of Web service communities

- interactive execution of process diagrams

- support for execution of generic operations invoked from BPEL processes

Due to its facilities, WSC system can be used in organizations and institutions that need to organize their own services in semantic communities, so that their business partners may get easy access to these services.

\section{Semantic Communities, Communities Taxonomy}

A community is specific for an interest domain and is created by a consortium of organizations (community provider) that wants to standardize Web services used in that domain. A community defines a generic operation collection for an area of interest, which Web services from that area have to implement. Community providers create descriptions of their communities using WSDL-S format (WSDL format extended with semantics ) Semantic attributes used in the WSDL-S representations are defined in the Community ontology and they describe domain, generic operations, input/output messages, the eligibility requirements, order for invoking operations, conditions for chaining operations etc.

The descriptions of communities, in WSDL-S format, are stored in the community register (CR) that is modeled as a taxonomy of communities based on specialization relationship between community domains. Web service providers describe their services in WSDL-S format according to Service ontology and transmit this description to WSC system to be stored in the repository service. Descriptions of communities and Web services are used by the system or administrator to register Web services with the communities.
In order to access Web services registered with a given community $(\mathrm{C})$, the user invokes a target operation $(\mathrm{O})$ of $\mathrm{C}$, using the graphic interface of WSC system. The system will generate, in a recursive way, the process diagram attached to operation $\mathrm{O}$. The process diagram is a graphical tree having as root the generic operation $O$. The level 1 of tree contains the generic operations to be performed before the operation $\mathrm{O}$. The level $\mathrm{n}$ of tree contains the generic operations to be performed before operations on level $n-1$.

The user executes interactively process diagram, starting with final nodes and finishing with the root node of tree. To execute a node operation, the user selects a Web service registered with the community $\mathrm{C}$ and then invokes the Web service method that corresponds to node operation. Execution state of each node of process diagram is stored in variables defined in the community $\mathrm{C}$.

Not only the user can access the Web services registered with communities of taxonomy, BPEL processes can also invoke generic operations of a community $\mathrm{C}$.

In this case, the system selects a Web service registered with community $\mathrm{C}$ and invokes the appropriate method of selected services.

\section{Example}

For example consider community $\mathrm{C}$ with the domain "Sporting Competition" and generic operations "RegisteringParticipant", "GettingMedicalApproval",

"ParticipatingCompetition".

To attend a sporting event, a user connects to the WSC system, selects from communities taxonomy the community $\mathrm{C}$ and then invokes generic operation "ParticipatingCompetition" of community C.

As a result of this invocation the system generates the process diagram as below:

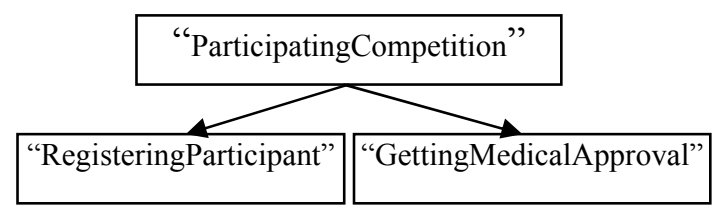

Then, the user interactively executes the generated diagram as follows: 
1. user clicks on generic operation "RegisteringParticipant" providing his personal data in the input variable "informatiiPersonale"; if the operation ends successfully it will return the participant's ID in the output variable " $u$ serId"

2. user clicks on generic operation "GettingMedicalApproval" in order to obtain medical approval, input variable "informatiiPersonale" already contains the user's personal data; if operation ends successfully it will return the medical approval in the output variable "avizMedical" will return the T-shirt number in output variable "nrtricou"

Execution of each operation in the above diagram involves checking the eligibility and termination conditions of the operation.

Description of community $\mathrm{C}$ from given example, using WSDL-S format, is illustrated in the Figure 1.

The above description contains concepts defined in Community ontology: function, synonym, domain, relation, precondition, predecessor, goal etc.

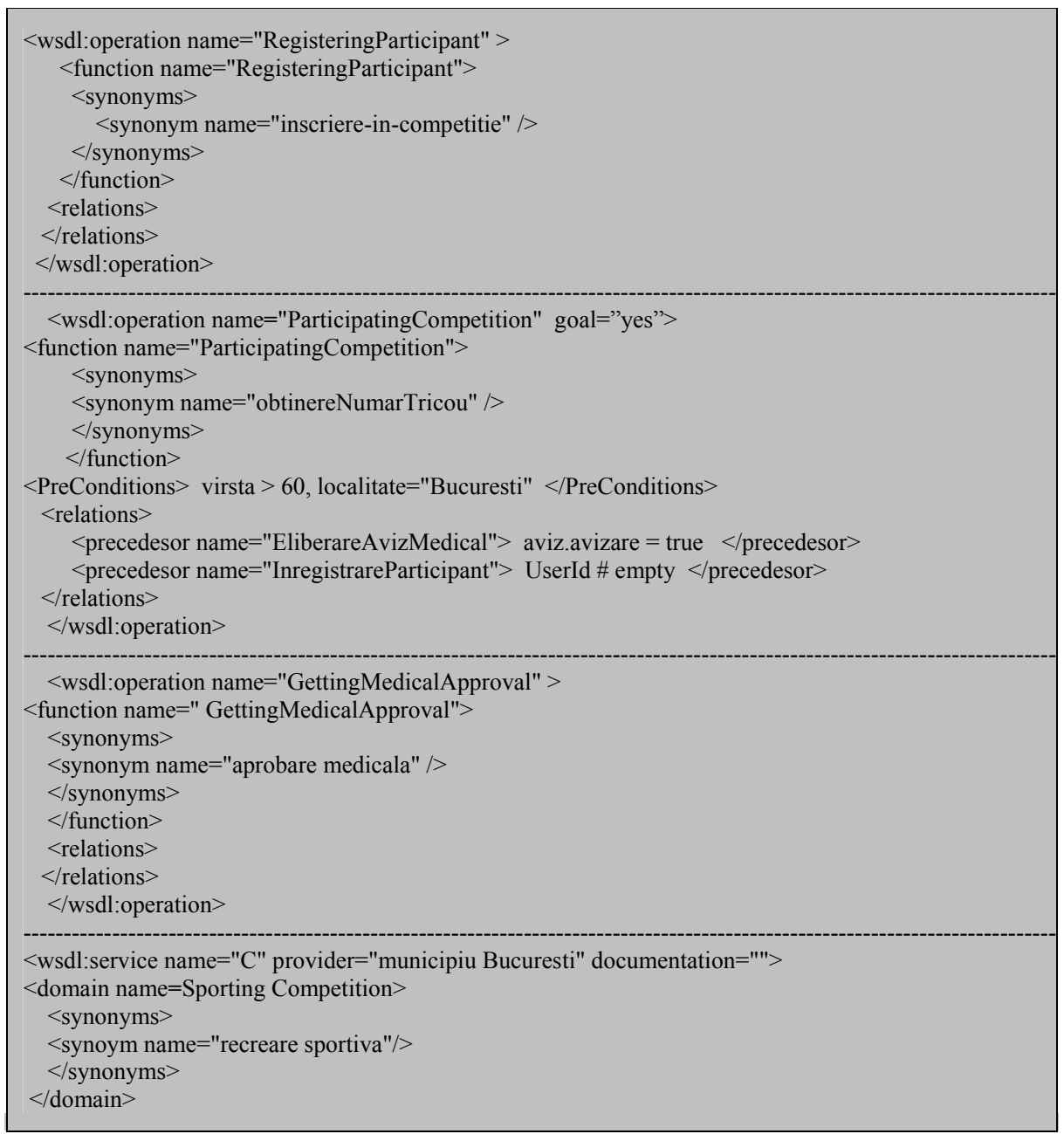

Figure 1. Description of Web services community for the given example

3. user clicks the generic operation "ParticipatingCompetition" to obtain his T-shirt number for participation at competition; input variables "userId" and "avizMedical" already contain the necessary information if the first operations ended successfully. If operation execution ends successfully it
The element <function> and synonyms included specify operation category, helping user to understand the operation semantics.

The element <domain> and synonyms included specify community domain, helping users to understand the semantics of domain of community. 
The element $<$ preconditions $>$ specifies the eligibility conditions for an operation to be invoked. Specified conditions include the input variables of the operation or variables defined in the user profile.

The element <relation $>$ specifies for each operation, the predecessor operations and their termination conditions. The termination conditions will include output variables of the operation.
Input/output variable of generic operations share the same memory area (global variables) if they have the same identifier, the same data type, and the same semantics. For example variable "userId" defined in the operation "RegisteringParticipant" and "userId" variable defined in the operation "ParticipatingCompetition" shares the same memory area, because they have the same

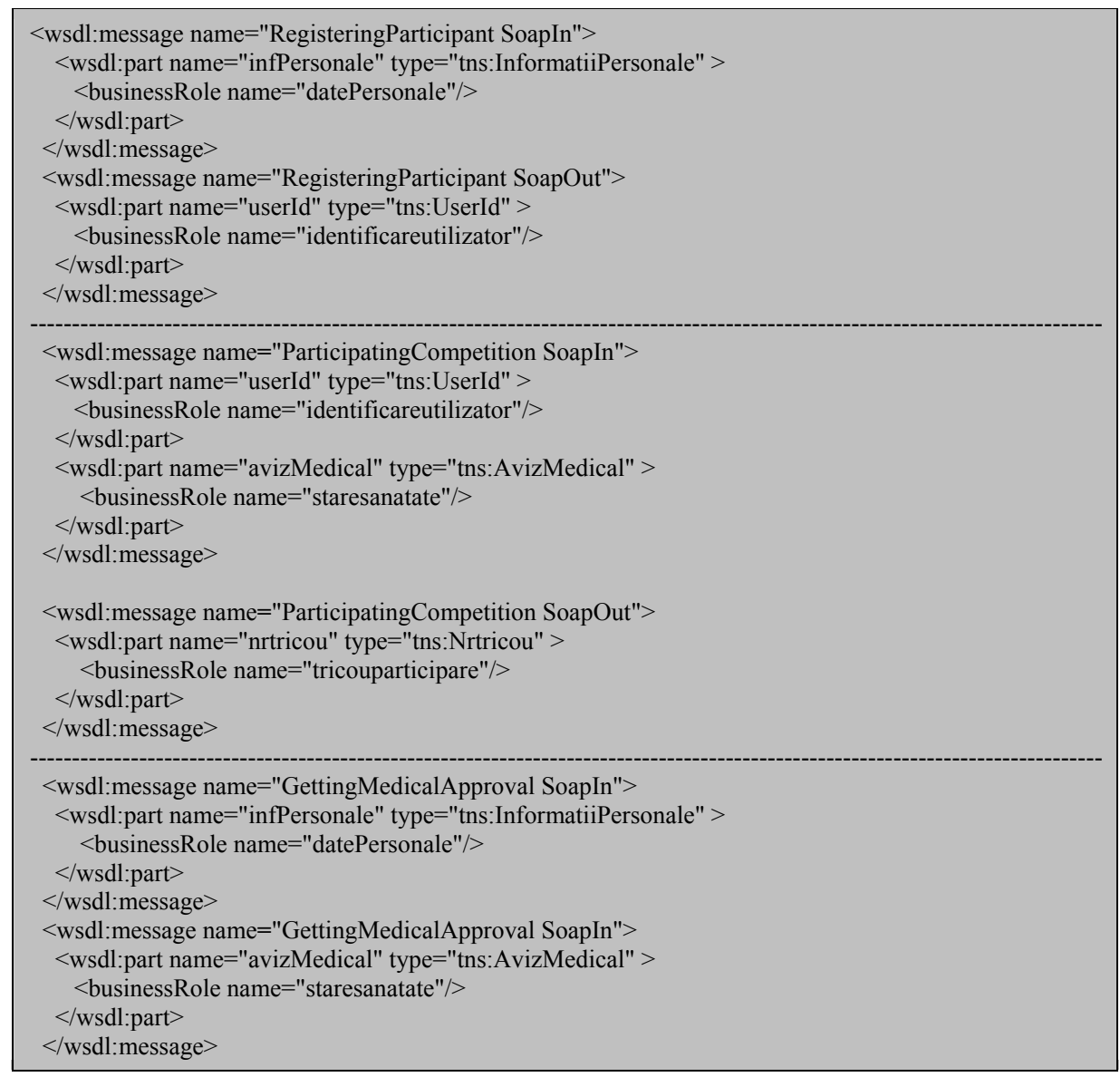

Figure 2. Description of input/output variables for generic operations

The attribute goal specifies whether an operation is a target operation or a helping operation. In the given example only operation "ParticipatingCompetition" is a target operation; the other operations are helping operations.

Description of input/output variable for the generic operations from given example is illustrated in the Figure 2.

Note that each input/output message is composed of several parts (variables), each part having attached a data type and a businessRole specifying semantics of variable. identifier, the same data type and the same businessRole.

Using global variables in describing communities allows interconnection among communities operations. The interactive execution of generic operations involves some editing of the input variable or viewing of the output variables.

The system WCS uses data type of each input/output variable to generate automatically editing and viewing controls. 
Data types for input/output variables used in the given example are defined in the Figure 3, using the XMLSchema formalism.
1. create the appropriate class having the same name as community's domain and

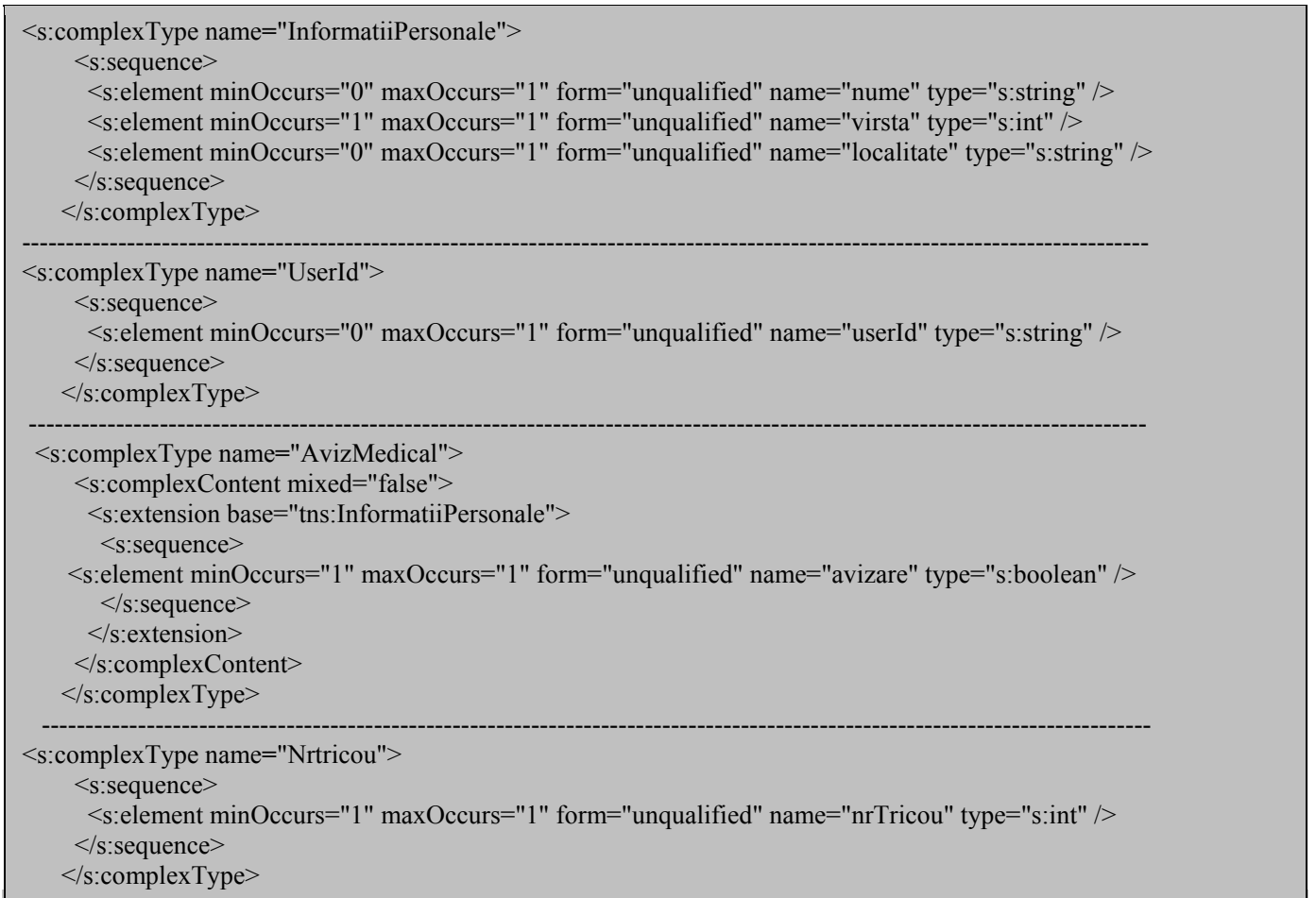

Figure 3. Data types used in the given example

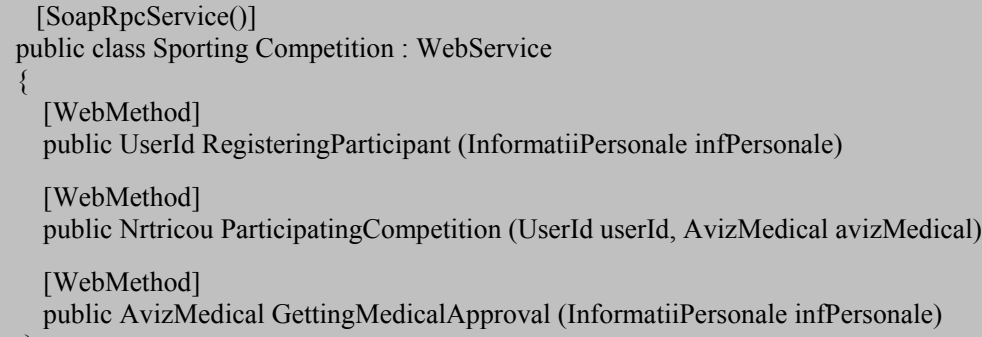

Figure 4.

\section{Note}

Data types used in WSC are only user types. If you want to use a system type, it must be incorporated into a user type.

For example, if the user needs an array of medical approval, then the system type "[]" will be incorporated into class "AvizMedicalArray" as below:

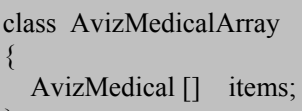

Manual creation of the file containing community description for given example is difficult. To ease this task we can use the following procedure: the methods identical with community operations - Figure 4 .

2. create the WSDL file format for this class using WSDL.exe instrument of .Net platform

3. manually extend WSDL file format with semantic attributes specified in Community ontology (domain, function, synonym, etc.)

4. modify variables name, created by the WSL tool, if needed (only output variables)

The value of "URL" attribute, from the obtained file, has to refer the community site. The community site allows execution of 
community operations when they are invoked from BPEL processes.
InsertCommunity that displays the insertion panel, as is illustrated in Figure 5.

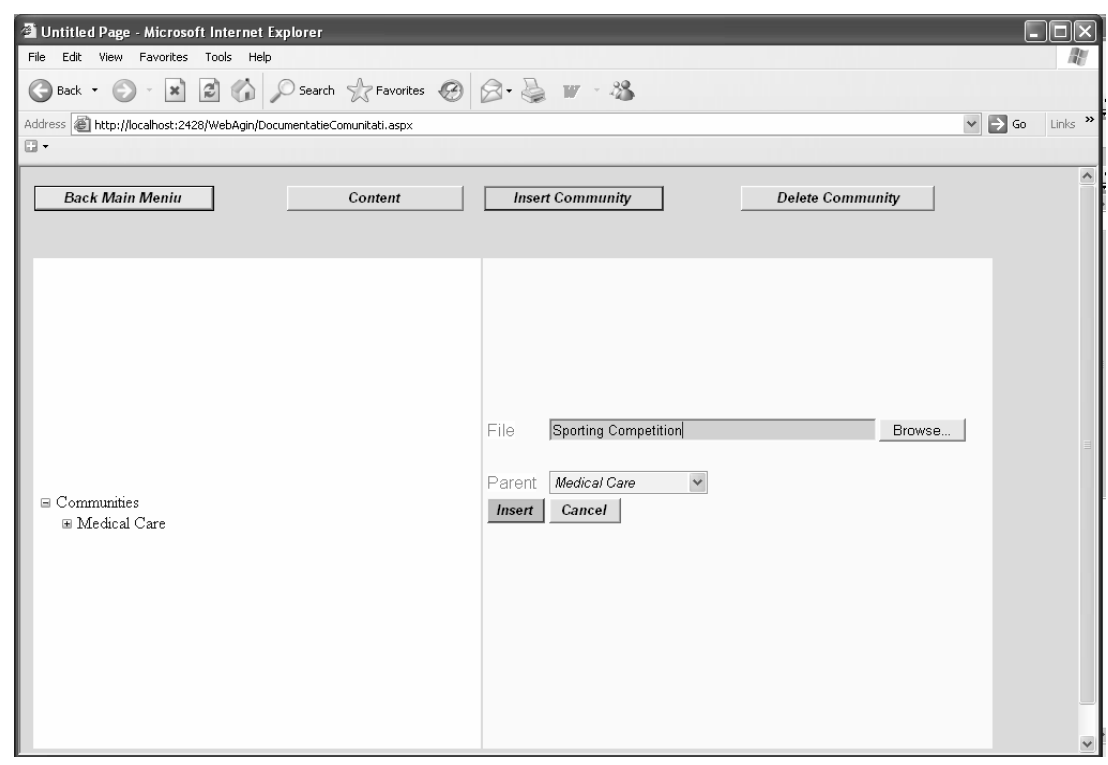

Figure 5. Insertion of a new community description in taxonomy

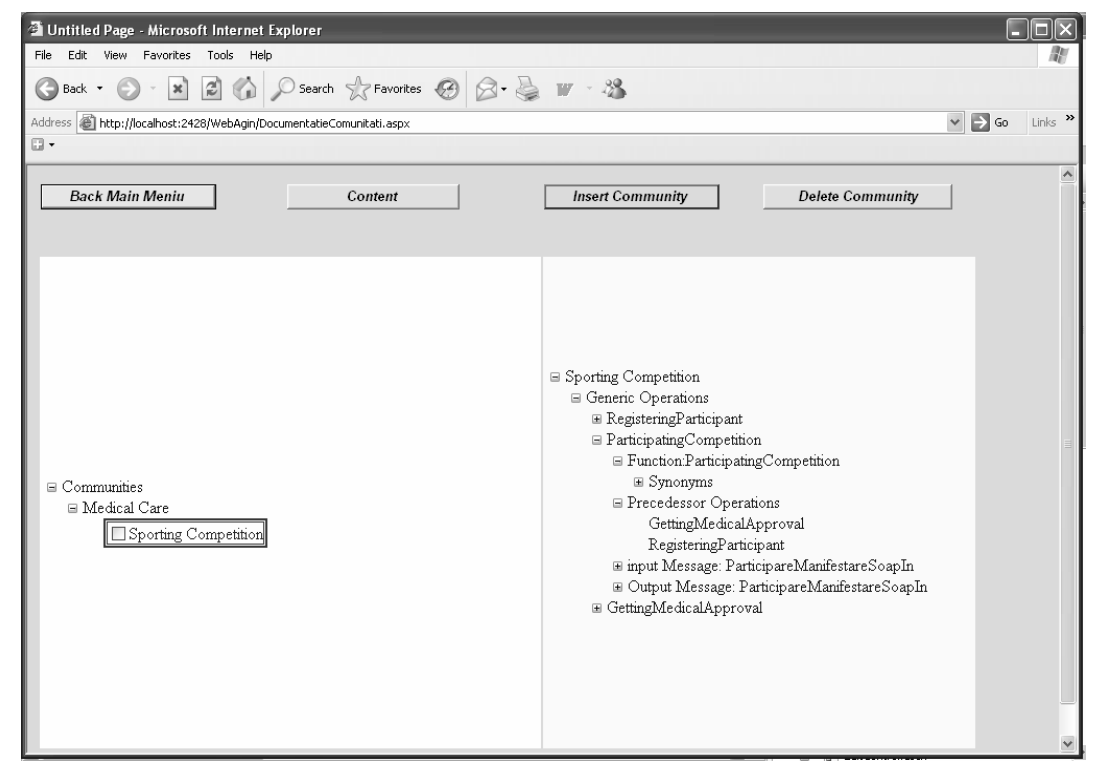

Figure 6. Visualization of the community's content

The file, thus obtained, will be parsed by WSC system to record the community description in the taxonomy of communities.

\subsection{User interface for managing communities}

WSC system provides communities management tool for insertion, deletion, navigation and display of community descriptions. [6] [7]

To insert a community description into the taxonomy, the user presses the button
The user specifies the place in taxonomy where new description will be inserted, by selecting from Parent list the parent community, then enters the WSDL-S file address in File box, which contains the description of community to be inserted, or uses Browser button to select the file containing community description. Actual insertion is done by pressing Insert button; the system will parse the specified file, will make a series of validations on the syntax and semantics of information and will build data structures necessary to record the new community description. 
In order to view the content of a community description, the user expands Communities tree, then clicks the label of community to select the community, then clicks Content button to list the content. The Figure 6 visualizes the content of the community given in the example.

Deleting a community from communities taxonomy can be done only by its supplier. To delete a community from the taxonomy, the supplier marks it by clicking community square, and then presses the DeleteCommunity button.

\section{Modeling and Registering Web Services}

Services implement generic operations defined in one or more communities. Service providers describe their services in WSDL-S files according to Service ontology. Semantic attributes defined in Service ontology are similar to those defined by the Community ontology with the following exceptions:

- Unlike Community ontology where all generic operations inherited the community domain, the Service ontology requires as every method of a Web service owns a domain, because a service can implement generic operations for several communities.

- The Service ontology does not define the eligibility conditions, termination conditions and sequence relationships between methods, but defines quality attributes for methods: cost, response time, reliability, confidentiality.

Using Service ontology, service providers create descriptions of services implemented by them and then send them to register with the appropriate communities. The registration process of services with semantic communities includes the following steps:

1. compare syntactic attributes of community operations with syntactic attributes of service methods (identifiers, data types etc.)

2. compare semantic attributes of community operations with semantic attributes of the methods services (domain, function, synonym, businessRole etc.)

As a result of registration procedure, a service (S) will be registered with a semantic community (C) if for some generic operations $(\mathrm{O})$ belonging to community $\mathrm{C}$, was found some methods (M) belonging to the service $\mathrm{S}$, so that the description of $\mathrm{M}$ fits the description of $\mathrm{O}$.

A Web service can be registered with one or several communities. [6] [7]

To illustrate the services modeling and registration method, we consider a Web service that implements all generic operations of community given in the above example. The WSDL-S file containing description of service methods is listed in Figure 7.

The description of input/output variables for each service method is done inside the $<$ element $>$ tag, corresponding to the method, not inside a message.

For example, input variables "userId" and "avizMedical" of "ParticipatingCompetition" method are defined in Figure 8.

For automating the WSDL-S file creation containing the service description we can use the following procedure:

1. create the appropriate service class having same methods as the service description - Figure 9.

2. create the WSDL file for this class, using WSDL.exe tool provided by .Net platform

3. extend the created WSDL file with the semantic attributes specified in the Service ontology (domain, function, synonym etc.)

4. change the name of variables in the created file, if it is necessary 


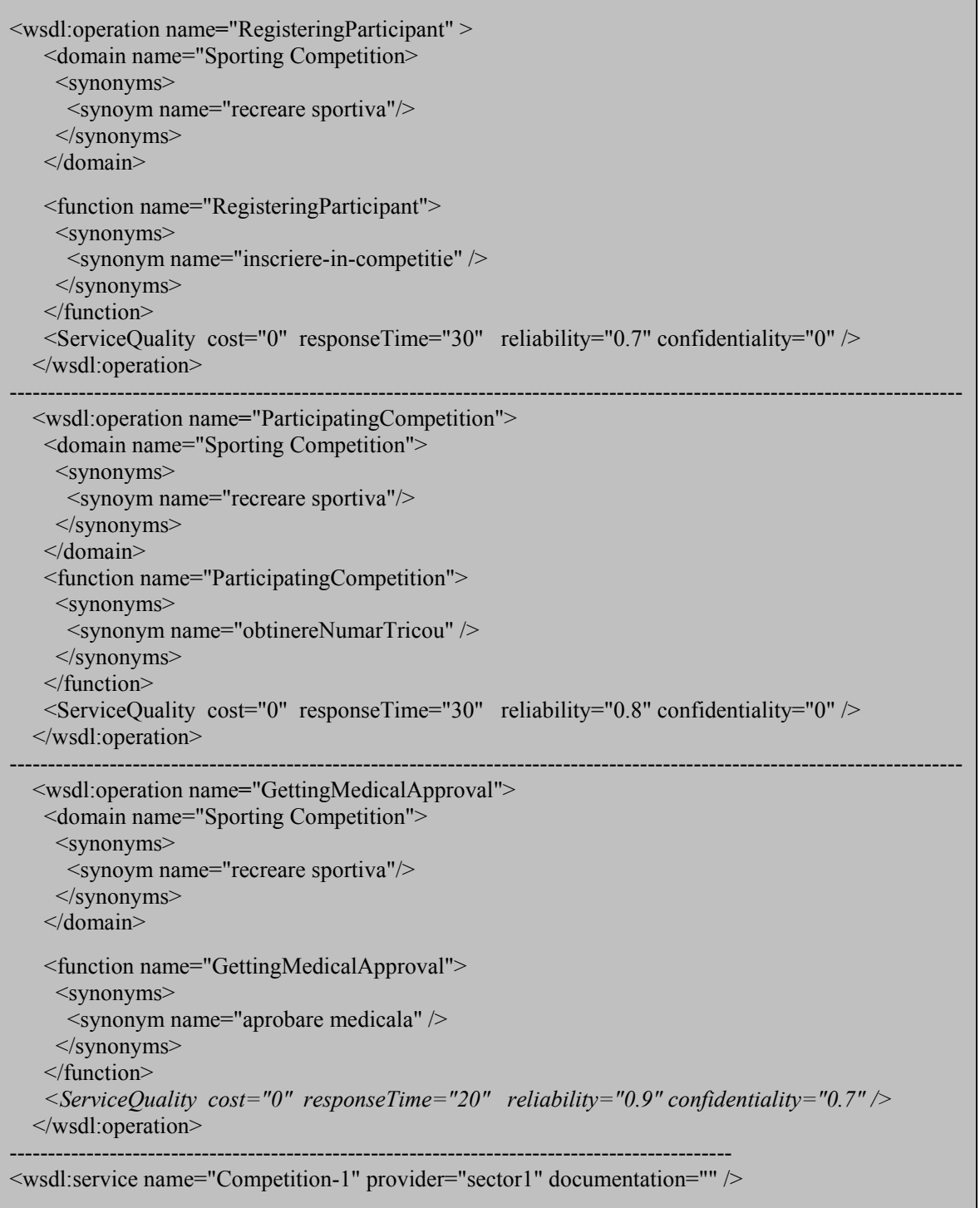

Figure 7. Description of the service's methods

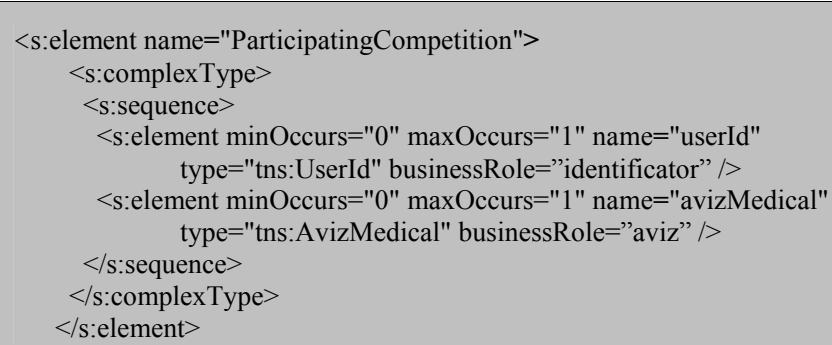

Figure 8. Description of service's variables

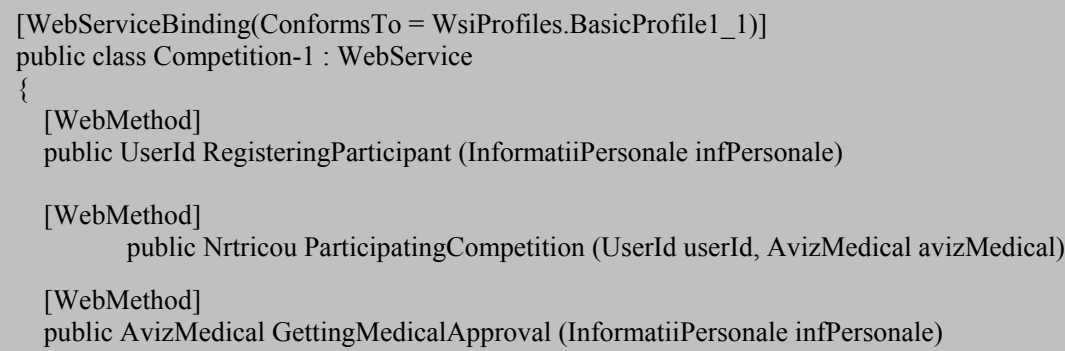

Figure 9. 
The file thus obtained, will be parsed by the WSC system to store the service description in repository and register the service with communities from taxonomy. order to parse, validate and insert the new description. The system will assign a unique identifier for each inserted description. Figure 10 shows the insertion panel.

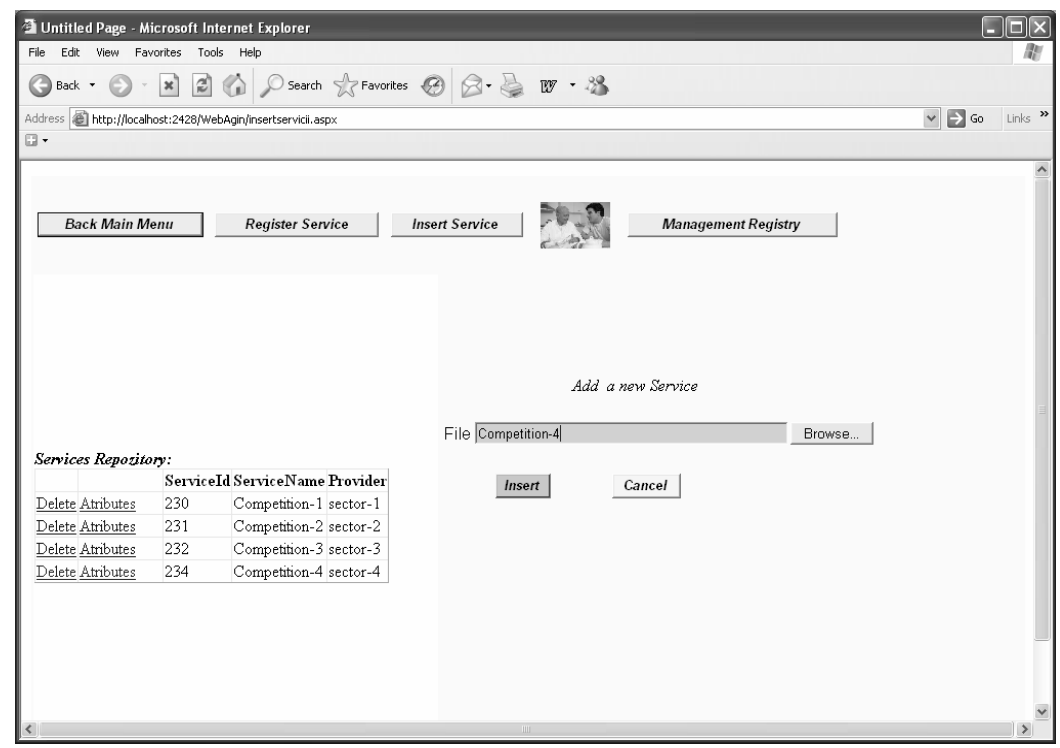

Figure 10. Insert a new Web service description

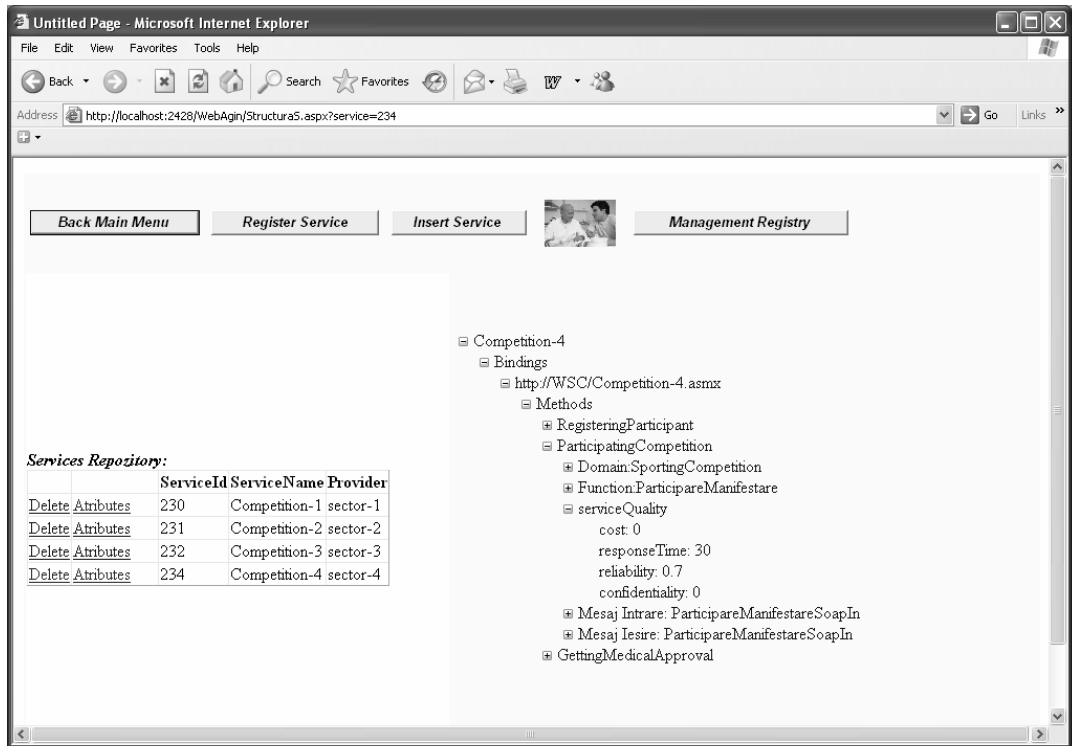

Figure 11. Visualization of service description attributes

\subsection{User interface for services management}

WSC system provides services management tool for inserting, deleting and viewing service descriptions. To display the insertion panel, the user clicks the Insert Service button. The user clicks the Browser button on the panel for selecting the WSDL-S file containing the description to be inserted. Then, the user presses the Insert button in
To view attributes of a service description, the user clicks Attributes button corresponding to service description. Figure 11 shows attributes of services Competition-4.

To delete a service description from repository the user clicks the Delete button corresponding to service description.

Once inserted in the repository, a service description will be used to register it with the communities from taxonomy. The 
administrator can manually register the service with a community from taxonomy. For this purpose the administrator presses the Register Service button to display the registration panel - Figure 12.

Then, the administrator selects from the list Services the service (W) to be registered, and from Communities list the community (C) with which the service $\mathrm{W}$ will be registered. click on it). Box Operation will display selected operation.

- The administrator selects a method (M) fro the service $\mathrm{W}$, that fits operation $\mathrm{O}$ (doing click on it). Box Method will display the selected method.

- The administrator clicks Register button to create the pair $(\mathrm{O}, \mathrm{M})$ in registry.

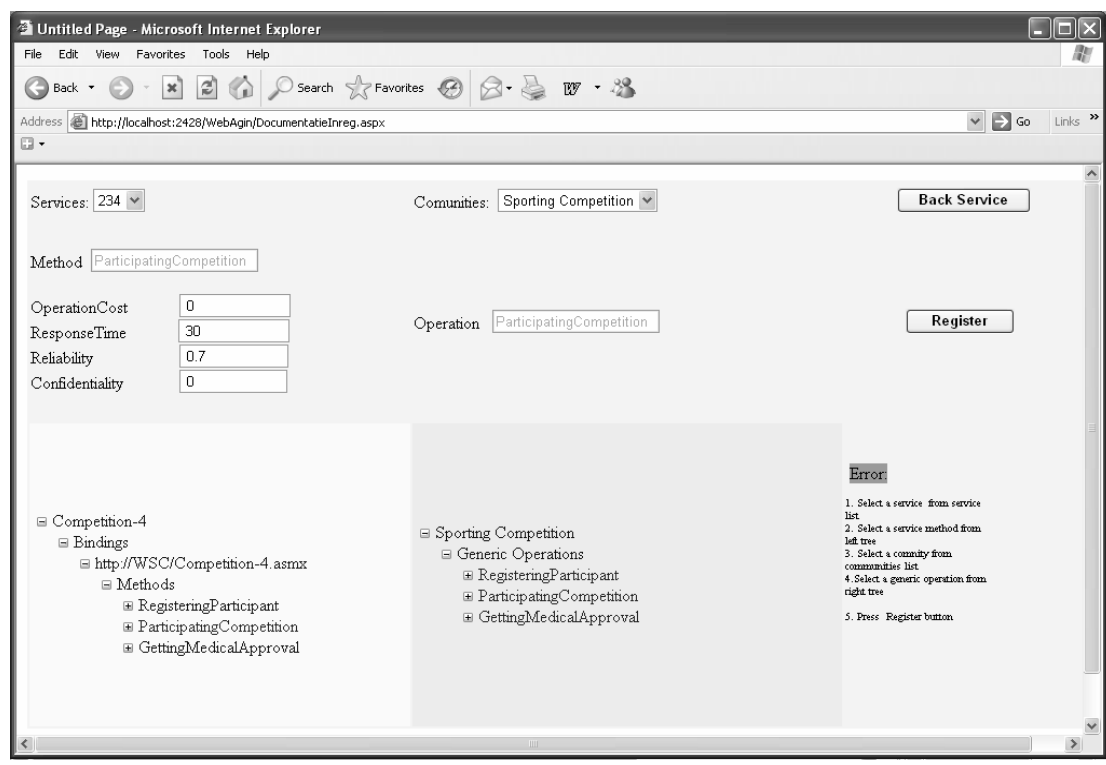

Figure 12. Registration of Web services with the communities

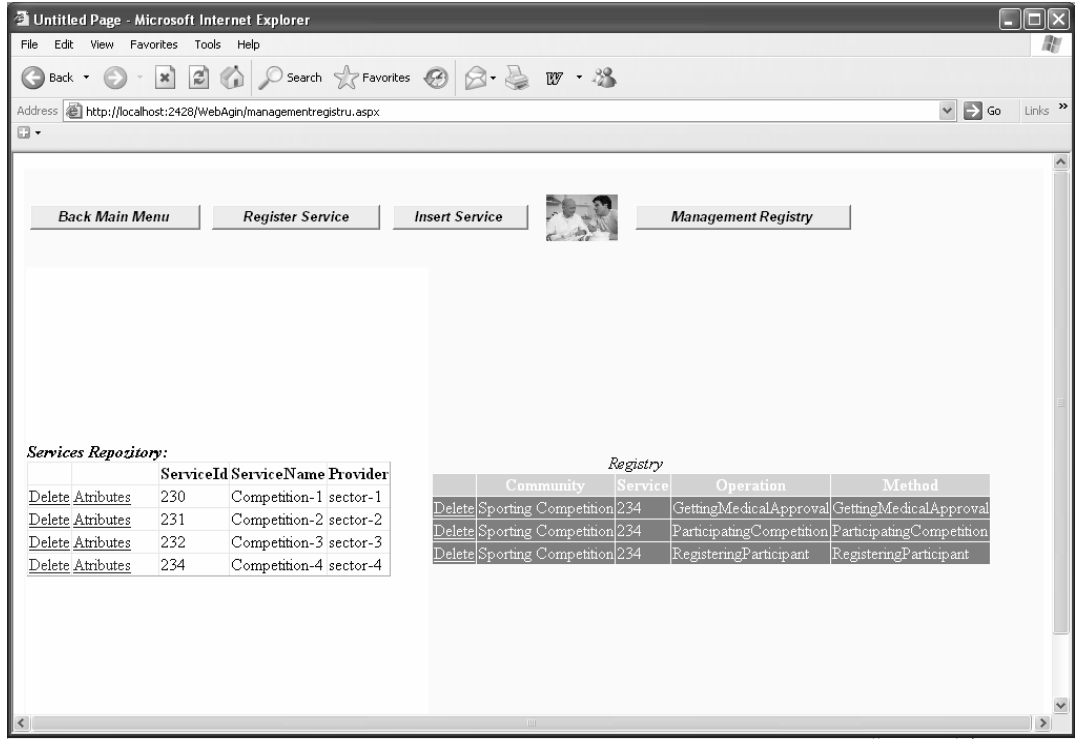

Figure 13. Visualization and deleting the registry

Next activity consists in mapping each generic operation of community $\mathrm{C}$ with the corresponding service method of service $\mathrm{W}$, as follows:

- The administrator selects a generic operation $(\mathrm{O})$ from community $\mathrm{C}$ (doing
The administrator can list or delete pairs from registry by clicking the Management Registry button. The grid containing existing entries from registry is displayed. To remove a pairs from registry the user clicks the Delete button corresponding to that pair - Figure 13. 


\section{Web Service Execution}

Execution of Web services registered with semantic communities is done as follows:

- the user selects a community from taxonomy

- the user invokes one of the target operation belonging to selected community

- the system recursively generates process diagram attached to operation invoked; the diagram includes all previous operations to be performed before the operation invoked

- the user interactively executes every node of the process diagram, starting with final nodes and finishing with the root node of the diagram as follows:

- selects a Web service from the services registered with the selected community, taking in account quality attributes of services

o initializes the input variables of the generic operation contained in the current node

o executes the service method corresponding to the generic operation contained in current node

- analyze output variables returned by the execution of method.

\subsection{User interface for Web services execution}

The user browses the taxonomy of communities, analyzing their semantic attributes for understanding semantic capabilities of communities. In the end he selects a community of interest and invokes a target operation from selected community. The system generates the process diagram attached to the invoked operation, the diagram is a tree having invoked operation as root, on the first level diagram includes all predecessors of invoked operation, on second level the diagram includes the predecessors of each operation belonging to the first level, and so on. [3], [4], [5]

The WSC system provides a user interface for navigation, selection and process diagram generation as is illustrate in Figure 14.

The user selected the community "Sporting Competition" as current community, clicking its label in taxonomy, then he listed the content of selected community by clicking the Content button, then he selected the target operation "ParticipatingCompetition" clicking its label in the content; the text box SelectedOperation will contain the selected operation.

Finally, the user clicked the Generate Process Diagram button to generate process diagram for invoked operation.

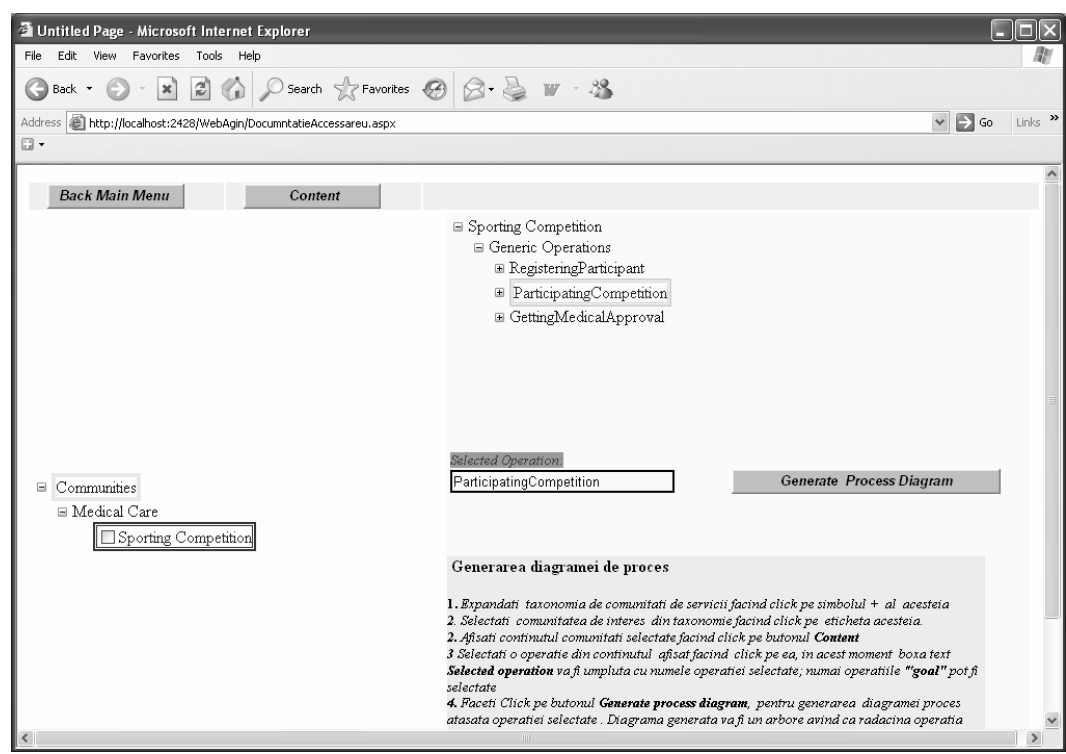

Figure 14. User interface for generating process diagram 
As result, the system generated the process diagram and displayed the execution panel, as shown in Figure 15. built by,system according to the data type of variable; for an uninitialized variable, the editing form contains value -1 for all

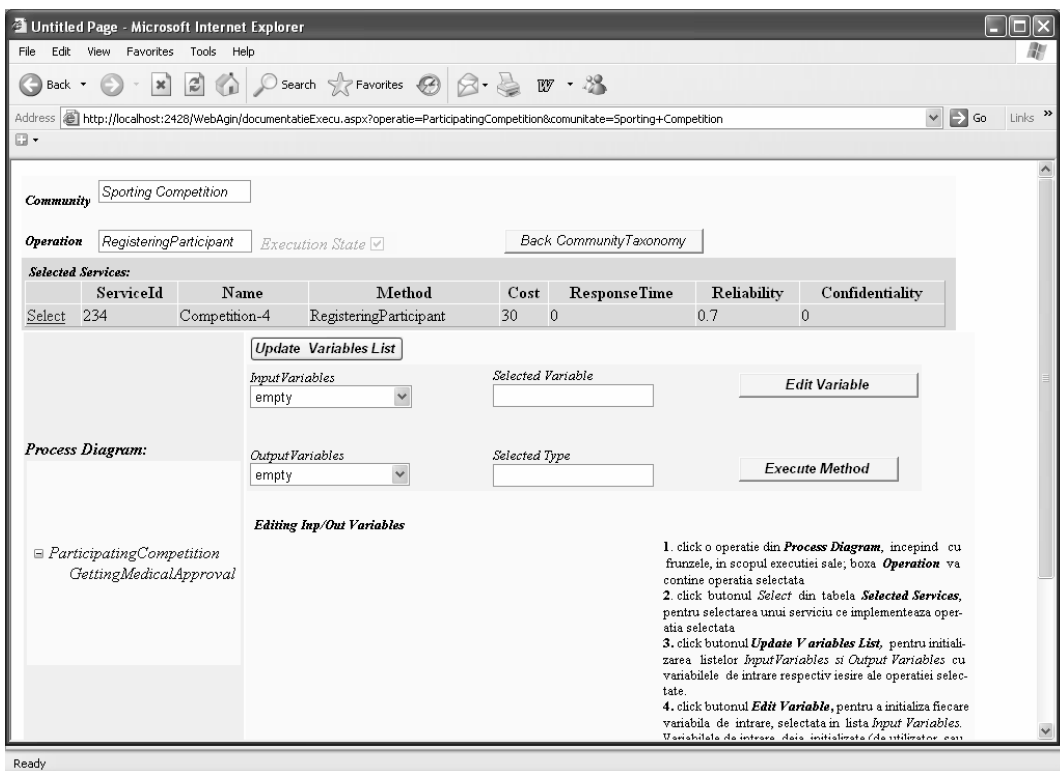

Figure 15. Execution panel for process diagrams

In our example, the generated process diagram has the root the operation "ParticipatingCompetition" and the first level contain the operations "GettingMedicalApproval" and "RegisteringParticipant".

To execute the generated process diagram, the user will execute each operation in the process diagram by: initializing the input variables of operation, selecting a service registered with the current community, executing the appropriate method of selected service, visualizing the output variables returned by executed method.

So, to execute the process diagram for our example, the user will perform:

1. Click on the operation "RegisteringParticipant" from diagram to select it; the box Operation will be filled automatically by the system with the selected operation.

2. Initialize the input variables of selected operation; for this purpose, the user selects from the list InputVariables the variable infPersonale. If it doesn't yet exist in the list, the Update Variable List button will be pushed. The user clicks EditVariable button to display the editing form. The editing form is automatically its fields. After the user enters personal data in the fields of form, he clicks Update button on form to initialize the variable with entered values.

3. Select a service registered with the current community by clicking the Select button on the table "Selected Services". The table contains for each method of a Web server registered with the current community the quality attributes of service.

4. Execute the appropriate method of selected service; the user presses the button Execute Method for executing the method of selected Web service. The system will perform the following steps:
assessment of eligibility conditions for operation "RegisteredParticipant";
invocation of service method;
assessment of termination conditions, at the end of method invocation;
filling the output variables with returned values

5. Visualize the output variables returned by method invocation; the user selects the output variable userId from OutputVariable list, then click the Edit Variable button to visualize it. 
If the eligibility conditions or termination conditions are not met, checkbox ExecutionState will become unmarked and diagram execution cannot continue.

After executing "RegisteredParticipant" operation, the system will delete it from process diagram and the user will go through the same process for executing the rest of diagram nodes.

\section{Composition of Communities Using the Language BPEL}

The description of a community contains the URL address for the website that implements the generic operations of that community.

The website redirects the invocation of each generic operation of community towards the invocation of a method of service registered with the community. The selection of a Web service from the services registered with the community, is done in accordance with website policy (default is selected service with lowest cost).

A website for a community also implements following methods:

- register for registering a service with the community

- selectService for selecting a service using a selection criterion

BPEL processes can use the community websites as partners, for this is necessary to know the WSDL files of the community websites. The order of invocation of generic operations for a given community, within a BPEL process, must comply with the order relation defined in the given community.

\section{Conclusions}

The WSC system was developed for organization, management and execution of Web services. The Web services are organized in semantic communities, all services registered with a given community have to implement all generic operations of given community. To access Web services, the users navigate through the taxonomy of communities, visualize the content of communities in order to understand the capabilities of service registered with them, and finally invoke the generic operations of communities.

Invocation of a generic operation can require execution of an entire process of preoperations, in this case the WSC system will generate process diagram for invoked operation and enable the interactive execution of this diagram.

Due to its facilities, the WSC system could be used to manage the Web services delivered through e-Romania portal[8].

\section{REFERENCES}

1. BRAHIM, M., A Dynamic Foundational Architecture for Semantic Web Services, Distributed and Parallel Databases, 17, 2005, pp. 179-206.

2. POPA, V., L. CONSTANTINESCU, C. ROTUNĂ, Senior Citizen Service Management using WebAging System, Studies in Informatics and Control, Volume 18, Issue 4, 2009.

3. RAN, S., A Model for Web Services Discovery with QoS, SIGecom Exchanges, vol. 4, no. 1, 2003.

4. MOISE, M., V. POPA, M. ZINGALE, L. CONSTANTINESCU, AL. PÎRJAN, WebAgeing - A Flexible System for Personalized Accessing of Services for Ageing Population, International Journal of Computers, Communications \& Control, Supplementary Issue Proceedings of ICCCC 2008, Vol. III, 2008, www.journal.univagora.ro.

5. MOISE, M., V. POPA, E-learning System Architecture for Personalized Publication and Access of Learning Resources, Using the Approach Based on Semantic Metadata, in Metalurgia International Journal Vol. XV, Special 
Issue, nr. 3, 2010, ISSN 1582-2214, pp. 207-210.

6. MEDJAHED, B., A. BOUGUETTAYA, A. ELMAGARMID. Composing Web Services on the Semantic Web. The VLDB Journal, Special Issue on the Semantic Web, September 2003.
7. OUZZANI, M., B. BOUGUETTAYA, Efficient Access to Web Services. IEEE Internet Computing, 37(3), March 2004.

8. SANDU, G., Achieving Modernization through ICT and New Technologies, Romanian IT\&C Directory - The $10^{\text {th }}$ Edition, March 2010. 